\title{
Clinical utility gene card for: Catecholaminergic polymorphic ventricular tachycardia (CPVT)
}

\author{
Carlo Napolitano ${ }^{\star, 1,2}$, Raffaella Bloise ${ }^{1}$, Mirella Memmi $^{1}$ and Silvia Giuliana Priori ${ }^{1,2,3}$ \\ European Journal of Human Genetics (2014) 22, doi:10.1038/ejhg.2013.55; published online 3 April 2013
}

\section{DISEASE CHARACTERISTICS}

1.1 Name of the disease (synonyms)

Synonyms: Familial polymorphic Ventricular ${ }^{1-9}$ Tachycardia (FPVT), catecholamine-induced polymorphic ventricular tachycardia (CPVT). Includes: RYR2-related catecholaminergic ventricular tachycardia, CASQ2-related catecholaminergic ventricular tachycardia, and catecholamine-sensitive polymorphic VT.

\subsection{OMIM\# of the disease}

604772, 611938

1.3 Name of the analysed genes or DNA/chromosome segments RyR2, cardiac ryanodine receptor; CASQ2, cardiac calsequestrin.

\subsection{OMIM\# of the gene(s)}

180902,114251

Two genes are clearly associated with CPVT (RyR2 autosomal dominant and CASQ2 autosomal recessive). Triadin and calmodulin mutations have been shown in possible CPVT cases and in one family with stress-induced sudden death. In the clinical setting, the analysis of these two genes may be indicated (yield yet unknown) when $R y R 2$ and CASQ2 screening is negative.

\subsection{Mutational spectrum}

Mutations are mainly missense. Exon 3 deletion is reported in two families with exercise-induced arrhythmias but also non-typical CPVT arrhythmias (sinoatrial node and atrioventricular node dysfunction, and atrial standstill) and dilated cardiomyopathy.

\subsection{Analytical methods}

Direct DNA sequencing.

\subsection{Analytical validation}

Sequencing of both strands. When a mutation is identified, one should validate the mutation in an independent experiment by direct sequencing using a freshly prepared DNA dilution.

\subsection{Estimated frequency of the disease}

(incidence at birth ('birth prevalence') or population prevalence)

Prevalence is estimated in 1:10000 but robust estimates are unavailable.
1.9 If applicable, prevalence in the ethnic group of investigated person

No data available.

\subsection{Diagnostic setting}

\begin{tabular}{lll}
\hline & Yes & No \\
A. (Differential) diagnostics & $\bigotimes$ & $\square$ \\
B. Predictive Testing & $\bigotimes$ & $\square$ \\
C. Risk assessment in relatives & $\bigotimes$ & $\square$ \\
D. Prenatal & $\bigotimes$ & $\square$ \\
\hline
\end{tabular}

Comment:

Prenatal testing is available when mutation in the family is known.

\section{TEST CHARACTERISTICS}

\begin{tabular}{|c|c|c|c|c|}
\hline & \multicolumn{2}{|c|}{ Genotype or disease } & \multirow{2}{*}{$\begin{array}{l}\text { A: True positives } \\
\text { B: False positives }\end{array}$} & \multirow{2}{*}{$\begin{array}{l}\text { C: False negative } \\
\text { D: True negative }\end{array}$} \\
\hline & Present & Absent & & \\
\hline \multicolumn{5}{|l|}{ Test } \\
\hline \multirow[t]{2}{*}{ Positive } & A & B & Sensitivity: & $A /(A+C)$ \\
\hline & & & Specificity: & $D /(D+B)$ \\
\hline \multirow[t]{2}{*}{ Negative } & C & $\mathrm{D}$ & Positive predictive value: & $A /(A+B)$ \\
\hline & & & Negative predictive value: & $\mathrm{D} /(\mathrm{C}+\mathrm{D})$ \\
\hline
\end{tabular}

2.1 Analytical sensitivity

(proportion of positive tests if the genotype is present)

Close to $100 \%$ with direct sequencing. No cases of exon skipping are reported. Preferential amplification of one allele can happen when one of the primers is located on an SNP. This problem is avoided with careful primer design.

\subsection{Analytical specificity}

(proportion of negative tests if the genotype is not present) Close to $100 \%$.

\subsection{Clinical sensitivity}

(proportion of positive tests if the disease is present)

The clinical sensitivity can be dependent on multiple factors such as age or family history. In such cases a general statement should be

${ }^{1}$ IRCCS Fondazione Maugeri, Pavia, Italy; ${ }^{2}$ Cardiovascular Genetics, Leon Charney Division of Cardiology, New York University, New York, NY, USA; ${ }^{3}$ Department of Molecular Medicine, University of Pavia, Pavia, Italy

*Correspondence: Professor C Napolitano, Molecular Cardiology, IRCCS Fondazione Msugeri and New York University, Via Maugeri 10/10A, Pavia 27100, Italy. Tel: +39 0382592 050; Fax: +39 0382592 094. E-mail: carlo.napolitano@fsm.it 
given, even if a quantification can only be made case by case. In general, for patients with an established clinical diagnosis of typical CPVT the percentage of positive genetic tests is $70-80 \%$ (that is, only a minority of patients seem to carry mutation in unknown genes).

Penetrance of CPVT is estimated in $60-70 \%$.

\subsection{Clinical specificity}

(proportion of negative tests if the disease is not present)

The clinical specificity can be dependent on variable factors such as age or family history. In such cases a general statement should be given, even if a quantification can only be made case by case.

Likely to be higher than 95\% (5\% of 'silent' variants) as per personal experience. Robust literature data are not available.

\subsection{Positive clinical predictive value}

(lifetime risk to develop the disease if the test is positive)

Approximately $80 \%$ of patients become symptomatic up to 40 years if left untreated. Approximately $30 \%$ of genetically affected subjects experience cardiac arrest/sudden death in the absence of appropriate therapy.

\subsection{Negative clinical predictive value}

(Probability not to develop the disease if the test is negative) Assume an increased risk based on family history for a non-affected person. Allelic and locus heterogeneity may need to be considered.

Index case in that family had been tested:

$>90 \%$

Index case in that family had not been tested: $>90 \%$

\section{CLINICAL UTILITY}

\section{1 (Differential) diagnostics: The tested person is clinically} affected

(To be answered if in 1.10 'A' was marked)

Not applicable.

\subsubsection{Can a diagnosis be made other than through a genetic test?}

\begin{tabular}{ll}
\hline No & $\square$ (continue with 3.1.4) \\
Yes & Clinically \\
& Imaging \\
& Endoscopy \\
& Biochemistry \\
& Electrophysiology \\
& Other (please describe)
\end{tabular}

\section{Comment:}

CPVT diagnosis requires the demonstration of the typical cardiac arrhythmias during exercise stress test and/or Holter monitoring. There are no structural abnormalities of the heart. The phenotype can be mimicked in some cases with ARVC (arrhythmogenic right ventricular cardiomyopathy) but in this case there is fibrofatty infiltration and anatomical abnormalities in the right ventricle (abnormal eco and MRI). Patients with long QT syndrome variant 7 (also called Andersen-Tawil syndrome) due to KCNJ2 mutations may have a similar pattern of arrhythmias.

\subsubsection{Describe the burden of alternative diagnostic methods to the patient}

Resting electrocardiogram (usually normal), exercise stress test, Holter monitoring, echocardiography, and magnetic resonance. Electrophysiological study is not indicated for typical CPVT cases but it may help differential diagnosis in some patients with non-typical presentation and suspect of catecholamine-sensitive RVOT tachycardia or arrhythmogenic right ventricular tachycardia.

\subsubsection{How is the cost effectiveness of alternative diagnostic methods} to be judged?

When a disease-causing mutation is identified in the index patient, genetic testing can be offered to apparently healthy relatives within the family to determine whether they carry the same mutation and are at risk of developing the disease. If the relative carries the mutation, a therapy is always indicated even in asymptomatic subjects without signs of the disease, given the risk of sudden death as first manifestation of the disease. All mutation carriers should be treated lifelong with non-selective beta blockers (propranolol or nadolol). Flecainide can be added for non-responders. The achievement of a satisfactory therapeutic effect can be assessed with exercise-stress test in the majority of cases. Regular medical follow-up is required to reassess the effective control of cardiac arrhythmias. Specific advice to mutation carriers might include restriction of physical activity (arrhythmias are triggered by catecholamine release), avoidance of specific careers and reproductive counseling.

If a relative does not carry the mutation then they can be reassured and cardiological follow-up discontinued (provided that severity of the phenotype in any family member does not suggest multiple genetic events).

\subsubsection{Will disease management be influenced by the result} of a genetic test?

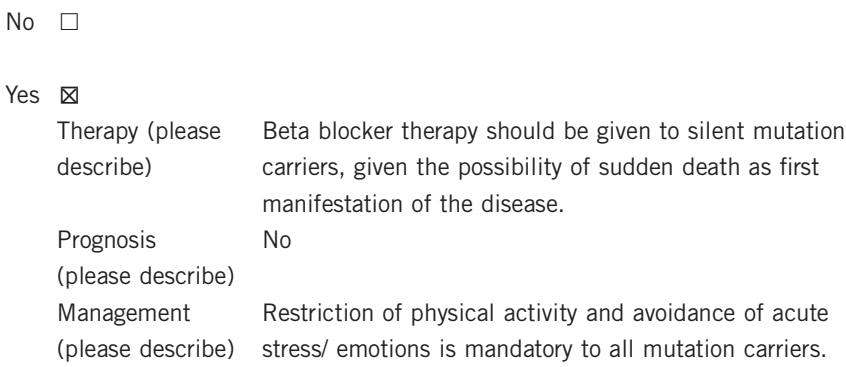

3.2 Predictive setting: The tested person is clinically unaffected but carries an increased risk based on family history

(To be answered if in 1.10 'B' was marked)

\subsubsection{Will the result of a genetic test influence lifestyle and} prevention?

If the test result is positive (please describe):

Avoidance of physical activity is indicated for all mutation carriers (both with and without phenotype).

If the test result is negative (please describe):

Subjects with clinical diagnosis and no mutations on the known genes should be treated and managed as those with a mutation.

3.2.2 Which options in view of lifestyle and prevention does a person at-risk have if no genetic test has been done (please describe)?

The same as with a positive genetic test.

3.3 Genetic risk assessment in family members of a diseased person (To be answered if in 1.10 ' $\mathrm{C}$ ' was marked) 
3.3.1 Does the result of a genetic test resolve the genetic situation in that family?

Predictive genetic testing of relatives is only possible if a pathogenic mutation has been identified in an affected person in the family. Autosomal dominant inheritance implies a 50\% risk of being a carrier of the pathogenic mutation in first-degree relatives of a person with the disease.

3.3.2 Can a genetic test in the index patient save genetic or other tests in family members?

Negative genetic testing in the proband cannot exclude the presence of the disease. Clinically unaffected children of genetically unaffected parents should not be tested.

3.3.3 Does a positive genetic test result in the index patient enable a predictive test in a family member?

Yes. All family members should undergo targeted-mutation(s) screening. All known genes should be entirely screened in the proband.

\subsection{Prenatal diagnosis}

(To be answered if in 1.10 'D' was marked)

3.4.1 Does a positive genetic test result in the index patient enable a prenatal diagnosis?

Yes.

\section{IF APPLICABLE, FURTHER CONSEQUENCES OF TESTING}

Please assume that the result of a genetic test has no immediate medical consequences. Is there any evidence that a genetic test is nevertheless useful for the patient or his/her relatives? (Please describe)
Even in the presence of clinically diagnosed and correctly treated CPVT in the proband, genetic testing is useful to identify at-risk family members.

\section{CONFLICT OF INTEREST}

The authors declare no conflict of interest.

\section{ACKNOWLEDGEMENTS}

This work was supported by EuroGentest2 (Unit 2: 'Genetic testing as part of health care'), a Coordination Action under FP7 (Grant Agreement Number 261469) and the European Society of Human Genetics.

1 Priori SG, Napolitano C, Tiso $\mathrm{N}$ et al: Mutations in the cardiac ryanodine receptor gene (hRyR2) underlie catecholaminergic polymorphic ventricular tachycardia. Circulation 2001; 103: 196-200.

2 Venetucci L, Denegri M, Napolitano C, Priori SG: Inherited calcium channelopathies in the pathophysiology of arrhythmias. Nat Rev Cardiol 2012; 9: 561-575.

3 van der Werf $\mathrm{C}$, Nederend I, Hofman $\mathrm{N}$ et al: Familial evaluation in catecholaminergic polymorphic ventricular tachycardia: disease penetrance and expression in cardiac ryanodine receptor mutation-carrying relatives. Circ Arrhythm Electrophysiol 2012; 5: 748-756.

4 Watanabe H, Chopra N, Laver D et al: Flecainide prevents catecholaminergic polymorphic ventricular tachycardia in mice and humans. Nat Med 2009; 15: $380-383$.

5 Hong RA, Rivera KK, Jittirat A, Choi JJ: Flecainide suppresses defibrillator-induced storming in catecholaminergic polymorphic ventricular tachycardia. Pacing Clin Electrophysiol 2012; 35: 794-797.

6 Priori SG, Napolitano C, Memmi M et al: Clinical and molecular characterization of patients with catecholaminergic polymorphic ventricular tachycardia. Circulation 2002; 10: 69-74.

7 Kimura H, Zhou J, Kawamura M et al: Phenotype variability in patients carrying KCNJ2 mutations. Circ Cardiovasc Genet 2012; 5: 344-353.

8 Faggioni M, Knollmann BC: Calsequestrin 2 and arrhythmias. Am J Physiol Heart Circ Physiol 2012; 302: H1250-H1256.

9 Priori SG, Chen SR: Inherited dysfunction of sarcoplasmic reticulum Ca2 + handling and arrhythmogenesis. Circ Res 2011; 108: 871-883. 